\title{
Quebras Estruturais e Estacionariedade da Razão Consumo- Renda: Novas Evidências para América Latina e Estados Unidos
}

\section{Structural Breaks and Stationarity of the Consumption-Income Ratio: New Evidence for Latin America and the United States}

\author{
Ana Cláudia Annegues da Silva* \\ Patrícia Soares de Araújo Carvalho** \\ Edilean Kleber da Silva Bejarano Aragón** *
}

\begin{abstract}
Resumo: Este trabalho investiga a estacionariedade da razão consumo-renda para 11 países da América Latina e os Estados Unidos no período de 1951 a 2010. Inicialmente, testa-se a existência de múltiplas quebras estruturais em datas desconhecidas na função tendência da razão consumo-renda. O teste utilizado, sugerido por Perron e Yabu (2009) e Kejriwal e Perron (2010), é robusto ao fato do componente de ruído da série temporal ser estacionário ou integrado. Em seguida, empregam-se os testes de raiz unitária propostos por Carrion-i-Silvestre et al. (2009), que permite múltiplas quebras no nível e na inclinação da função tendência. Os resultados indicam a presença de duas quebras na função tendência da razão consumo-renda para a maior parte das economias analisadas. As exceções são a Colômbia e o Paraguai, que apresentam apenas uma quebra. No caso dos Estados Unidos, a hipótese de ausência de quebra estrutural dos parâmetros da função tendência da propensão média a consumir não pode ser rejeitada. Com relação à ordem de integração da razão consumo-renda, os resultados indicam que essa variável é não estacionária para todos os países da amostra, com exceção do Peru.
\end{abstract}

Palavras-chave: Razão consumo-renda. Quebras estruturais. Testes de raiz unitária.

\begin{abstract}
This paper investigates the stationarity of the consumption-income ratio for 11 countries in Latin America and the United States in the period 1951-2010. Initially, it is tested the existence of multiple structural breaks at unknown dates in the trend function of consumption-income ratio. The test used, suggested by Perron and Yabu (2009) and Kejriwal and Perron (2010), is robust to the fact that the noise component of the time series is stationary or integrated. Then, it is employed the unit root tests proposed by Carrion-i-Silvestre et al. (2009), which allows for multiple breaks in the level and slope of the trend function. The results indicate the presence of two breaks in the trend function of the consumption-income ratio for most of the economies analyzed. The exceptions are Colombia and Paraguay, which have only one break. In the US case,

\footnotetext{
* Mestra em Economia pela Universidade Federal da Paraíba (UFPB). Doutoranda em Economia pela Universidade Federal do Rio Grande do Sul (UFRGS). E-mail: annegues.ana@gmail.com

** Doutoranda em Economia pela Universidade Federal da Paraíba (UFPB). Professora do Instituto Federal de Educação, Ciência e Tecnologia da Paraíba (IFPB).E-mail: patricia.araujo@ifpb.edu.br *** Doutor em Economia pela Universidade Federal do Rio Grande do Sul (UFRGS). Professor do Departamento de Economia e do Programa de Pós-Graduação em Economia da Universidade Federal da Paraíba (UFPB). E-mail: edilean@hotmail.com
} 
the hypothesis of no structural break in the trend function of the parameters of the average propensity to consume cannot be rejected. Regarding the order of integration of consumption-income ratio, the results indicate that this variable is not stationary for all countries in the sample, with the exception of Peru.

Keywords: Consumption-income ratio. Structural breaks. Unit root tests.

JEL Classification: C22; C12; E21.

\section{1 lntrodução}

A razão consumo-renda, ou propensão média a consumir (PMC), é um tema controverso na literatura econômica. De acordo com algumas teorias sobre o consumo agregado das famílias, o comportamento da PMC pode apresentar importantes diferenças. Por exemplo, a hipótese keynesiana da renda absoluta e a teoria marxista do subconsumo implicam uma razão consumo-renda não estacionária. Já a hipótese da renda relativa, a hipótese da renda permanente e a hipótese do ciclo da vida predizem uma relação de equilíbrio no longo prazo entre consumo e renda, ${ }^{1}$ ou seja, a razão consumo-renda é estacionária. Assim, os choques de política têm apenas efeitos temporários sobre a PMC. Em contraposição, uma PMC não estacionária mostra que choques afetam permanentemente a fração da renda destinada ao consumo.

Em termos empíricos, a literatura revela a existência de conflitos quanto à definição da ordem de integração da PMC. Algumas evidências indicam que a PMC é estacionária, enquanto outras mostram essa variável como sendo não estacionária. ${ }^{2}$ Inicialmente, esses resultados foram obtidos através de testes de raiz unitária convencionais, tal como o teste Dickey-Fuller aumentado (ADF).

Devido ao baixo poder do teste ADF, Sarantis e Stewart (1999) utilizam testes de raiz unitária para um painel de 20 países da Organização para Cooperação e Desenvolvimento Econômico (OCDE). Eles concluem que a razão consumo-renda para esses países é gerada por um processo estocástico não estacionário. Ao analisar 14 países europeus da amostra de Sarantis e Stewart (1999), Tsionas e Christopoulos (2002) utilizam testes de raiz unitária com ajustamento assimétrico e encontram evidências de estacionariedade da PMC em pelo menos um regime. Fallahi (2012) estuda a estacionariedade da PMC nos países da OCDE por meio da construção de intervalos de confiança para o parâmetro de persistência sob três diferentes procedimentos de bootstrapping. Cerrato et al. (2013) investigam a esta-

1 Sobre a hipótese da renda relativa, a hipótese da renda permanente e a hipótese do ciclo da vida, ver Duesenberry (1952), Friedman (1957) e Ando e Modiglinani (1963), respectivamente.

2 Drobny e Hall (1989), Molana (1991), Hall e Patterson (1992) e Horioka (1997) são alguns exemplos de trabalhos que produzem evidências da não estacionariedade da PMC. Por outro lado, Ungern-Stenberg (1986), Campbell (1987), King et al. (1991) e Slesnick (1998) mostram que a PMC é estacionária. 
cionariedade da $\mathrm{PMC}$ em 24 países da OCDE e 33 países não membros da OCDE. Para isso, eles utilizam testes de raiz unitária para painel não linear e concluem que a PMC foi não estacionária em mais de $70 \%$ dos países de ambos os grupos.

Enquanto vários testes têm sido sugeridos para verificar a estacionariedade da razão consumo-renda, uma menor atenção tem sido dada à presença de quebras estruturais nessa variável. Desde o trabalho de Perron (1989), sabe-se que a presença de quebras estruturais pode reduzir fortemente o poder dos testes de raiz unitária. Diante disso, Cook (2005) aplica o teste do Multiplicador de Lagrange ${ }^{3}$ (LM) mínimo de raiz unitária com quebras estruturais para a amostra de países analisada por Sarantis e Stewart (1999). Ao contrário desses autores, Cook (2005) encontra que a PMC foi estacionária para as economias estudadas. Gomes e Franchini (2009) utilizam os testes ADF e LM mínimo de raiz unitária com uma e duas quebra estruturais para verificar a ordem de integração da PMC nos países da América do Sul. Esses autores encontram evidências que mostram que a razão consumo-renda é estacionária.

O objetivo do presente trabalho é investigar as propriedades estocásticas da razão consumo-renda levando em consideração possíveis quebras estruturais nessa variável. Diferente da maioria dos trabalhos mencionados, que focam em economias da OCDE, analisa-se o comportamento dinâmico da PMC para 11 países da América Latina e também dos Estados Unidos. Os países latino-americanos constituem um interessante caso de estudo porque são mais voláteis e sujeitos a mudanças institucionais na condução da política econômica do que as economias desenvolvidas. Empiricamente, estende-se a análise de Gomes e Franchini (2009) ao se realizar a investigação em duas etapas. ${ }^{4}$ Primeiro, testa-se a existência de múltiplas quebras estruturais em datas desconhecidas no nível e na inclinação da função tendência da PMC. Isso é feito através da utilização do procedimento sequencial e da estatística de teste apresentada por Perron e Yabu (2009) e Kejriwal e Perron (2010). Esse teste não necessita de um conhecimento a priori se o componente de ruído da série temporal é estacionário ou integrado, além de fornecer estimativas consistentes das datas das quebras. Na segunda etapa, testa-se a ordem de integração da razão consumo-renda através de dois grupos de testes. Para os casos em que a PMC apresenta quebra estrutural na função tendência, utilizam-se os testes de raiz unitária propostos por Carrion-i-Silvestre et al. (2009). Esses testes admitem a presença de múltiplas quebras estruturais na função tendência sob ambas as hipóteses, nula e alternativa. Caso não haja quebra na função tendência, a classe de testes M de raiz unitária, analisada por Ng e Perron (2001), é empregada.

3 Do inglês Lagrange Multiplier.

4 Além de incluir México e Estados Unidos em um período mais recente (1951-2010), este trabalho amplia a análise de Gomes e Franchini (2009) ao realizar testes estatísticos específicos para verificar a existência de quebras estruturais nos coeficientes da função tendência e empregar três testes de raiz unitária. 
A realização de testes de raiz unitária com quebra estrutural apenas para as variáveis em que se rejeita a hipótese nula de ausência de quebras na função tendência é importante porque esses testes sofrem de baixo poder na ausência de quebras. ${ }^{5}$

Em geral, os resultados podem ser sumarizados da seguinte forma: primeiro, o teste para quebras estruturais na função tendência da PMC indicou a existência de duas quebras para a maior parte das economias analisadas - as exceções foram a Colômbia e o Paraguai, que apresentaram apenas uma quebra; segundo, para os Estados Unidos, não se pôde rejeitar a hipótese de ausência de quebras estruturais dos parâmetros da função tendência da PMC. Por fim, a análise da ordem de integração da razão consumo-renda mostrou que essa série temporal não é estacionária para todos os países da amostra, com exceção do Peru.

Além desta introdução, este trabalho está organizado em mais três seções: a segunda apresenta a metodologia econométrica; a terceira traz uma análise descritiva dos dados e revela os resultados empíricos; e a quarta traça as considerações finais.

\section{Procedimentos Empíricos}

Esta seção descreve os procedimentos metodológicos empregados na análise do comportamento dinâmico da PMC. Em primeiro lugar, faz-se uma breve apresentação do teste utilizado para verificar a existência de quebras estruturais na função tendência da razão consumo-renda. Em seguida, os testes de raiz unitária que permitem quebras estruturais na função tendência são descritos.

\subsection{Testes de Perron e Yabu (2009) e Kejriwal e Perron (2010) para Quebras na Tendência Determinística}

Para testar a presença de quebra estrutural na função tendência da razão consumo-renda, utiliza-se o procedimento de teste de Perron e Yabu (2009) e a sua extensão para múltiplas quebras, proposto por Kejriwal e Perron (2010). A vantagem desse procedimento é que permite testar mudanças estruturais no nível e na inclinação da função tendência de uma série temporal sem o conhecimento a priori sobre a ordem de integração do componente de ruído.

O teste para verificar a presença de quebra estrutural nos parâmetros da função tendência é baseado em um procedimento de mínimos quadrados generalizados quase factíveis (FGLS). ${ }^{6}$ Perron e Yabu (2009) supõem que o processo gerador dos dados para a variável aleatória $y_{t}$ é dado por:

\footnotetext{
5 Adicionalmente, Campbell e Perron (1991) mostram que a especificação adequada dos componentes determinísticos é importante para se obter testes de raiz unitária com propriedades confiáveis para pequenas amostras.

6 Do inglês quasi-feasible generalized least squares (PERRON; YABU, 2009).
} 


$$
\begin{aligned}
& y_{t}=\mathrm{x}_{\mathrm{t}}^{\prime} \psi+u_{t} \\
& u_{t}=\alpha u_{t-1}+v_{t} \\
& v_{t}=d(L) e_{t}
\end{aligned}
$$

para $t=1, \ldots, T$, em que $x_{t}$ é um vetor $(r \times 1)$ de componentes determinísticos, $\Psi$ é um vetor ( $r \times 1$ ) de parâmetros específicos, $d(L)=\sum_{i=0}^{\infty} d_{i} L^{i}, d(L)=\sum_{i=0}^{\infty} i\left|d_{i}\right|<\infty$ ,$d(1) \neq 0$ e $e_{t} \sim \operatorname{iid}\left(0, \sigma^{2}\right)$. Para o caso em que uma quebra no nível e na inclinação da função tendência ocorre em T1 $=[\lambda 1 \mathrm{~T}]$ para algum $\lambda_{1} \in(0,1)$, temos $x_{t}=(1$, $\left.D U_{t}, t, D T_{t}\right)$, com $D U_{t}=\mathrm{I}\left(t>T_{1}\right), D T_{t}=I\left(t>T_{1}\right)\left(t-T_{1}\right)$, em que I $(\cdot)$ é uma função indicadora. É importante destacar que $-1<\alpha \leq 1$, de modo que erros estacionários ou integrados são permitidos.

O procedimento de Perron e Yabu (2009) inicia-se com a estimação por mínimos quadrados ordinários (MQO) do coeficiente $\alpha$ presente na seguinte regressão:

$$
\hat{u}_{t}=\alpha \hat{u}_{t-1}+\sum_{i=1}^{k} \zeta_{i} \Delta \hat{u}_{t-i}+e_{t k}
$$

em que $k$ é escolhido pelo critério de informação bayesiano (BIC). Seja $\tilde{\alpha}$ a estimativa MQO de $\alpha$. Com a finalidade de aperfeiçoar as propriedades para a amostra finita, Perron e Yabu (2009) sugerem usar uma versão de $\tilde{\alpha}$ com correção de viés, $\tilde{\alpha}_{\mathrm{M}}$, para obter uma estimativa super eficiente de $\alpha$, dada por: ${ }^{7}$

$$
\widetilde{\alpha}_{M S}= \begin{cases}\widetilde{\alpha}_{M} & \text { se }\left|\widetilde{\alpha}_{M}-1\right|>T^{-1 / 2} \\ 1 & \text { se }\left|\widetilde{\alpha}_{M}-1\right| \leq T^{-1 / 2}\end{cases}
$$

em que

$$
\begin{aligned}
& \tilde{\alpha}_{M}=\tilde{\alpha}+C(\hat{\tau}) \hat{\sigma}_{\alpha}, \\
& C(\hat{\tau}) \begin{cases}-\hat{\tau} & \text { se } \hat{\tau}>\tau_{p c t} \\
I_{p} T^{-1} \hat{\tau}-(1+r)\left[\hat{\tau}+c_{2}(\hat{\tau}+a)\right]^{-1} & \text { se }-a<\hat{\tau} \leq \tau_{p c t} \\
I_{p} T^{-1} \hat{\tau}-(1+r) \hat{\tau}^{-1} & \text { se }-c_{1}^{1 / 2}<\hat{\tau} \leq-a \\
0 & \text { se } \hat{\tau} \leq-c_{1}^{1 / 2}\end{cases}
\end{aligned}
$$

em que $\hat{\tau}=(\tilde{\alpha}-1) / \tilde{\sigma}_{\alpha}, \tilde{\sigma}_{\alpha}$ é o desvio padrão de $\tilde{\alpha}, \mathrm{c}_{1}=(1+r) T$, r é o número de parâmetros estimados na função tendência, $c_{2}=\left[(1+r) T-\left(\tau_{\text {pct }}\right)^{2}\left(I_{p}+T\right)\right]\left[\tau_{\text {pct }}(a\right.$

$7 \quad$ Como destacado por Mariott e Pope (1954), Pantula e Fuller (1988) e Roy e Fuller (2001), o estimador $\mathrm{MQO}$ de $\alpha$ é viesado. 
$\left.+\tau_{\mathrm{pct}}(\mathrm{Ip}+\mathrm{T})\right]^{-1}$, a é alguma constante, $\tau_{\mathrm{pct}}$ é um percentil da distribuição limite de quando $\alpha=1, I_{p}=[(p+1) / 2]$ e $p$ é a ordem do processo autorregressivo. ${ }^{8}$

Perron e Yabu (2009) usam a estimativa $\tilde{\alpha}_{\mathrm{MS}}$ para construir a seguinte regressão quase diferenciada:

$$
\begin{gathered}
\left(1-\tilde{\alpha}_{M S} L\right) y_{t}=\left(1-\tilde{\alpha}_{M S} L\right) \mathrm{x}_{\mathrm{t}}^{\prime} \Psi+\left(1-\tilde{\alpha}_{M S} L\right) u_{t}, \quad t=2, \ldots, T \\
y_{1}=\mathrm{x}_{1}^{\prime} \Psi+u_{1}
\end{gathered}
$$

em que $\Psi=\left(\mu_{0}, \mu_{1}, \beta_{0}, \beta_{1}\right)^{\prime}$, e a estimativa resultante da equação 4 é $\tilde{\Psi}=\left(\tilde{\mu}_{0}^{F G}, \tilde{\mu}_{1}^{F G}, \tilde{\beta}_{0}^{F G}, \tilde{\beta}_{1}^{F G}\right)^{\prime}$.

Para testar a hipótese nula de ausência de quebra estrutural no nível e inclinação da função tendência $\left(H_{0}: \mu_{1}=\beta_{1}=0\right)$ na presença de erros $\mathrm{I}(0)$, o teste de Wald para uma dada fração de quebra $\lambda_{1}, W_{\mathrm{RQF}}\left(\lambda_{1}\right)$ é dado por:

$$
W_{R Q F}\left(\lambda_{1}\right)=[\mathrm{R}(\tilde{\Psi}-\Psi)]^{\prime}\left[\hat{h}_{\nu} \mathrm{R}\left(\mathrm{X} \mathrm{X}^{-1} \mathrm{R}^{\prime}\right]^{-1}[\mathrm{R}(\tilde{\Psi}-\Psi)]\right.
$$

em que $\mathrm{R}=\left(\begin{array}{cccc}0 & 1 & 0 & 0 \\ 0 & 0 & 0 & 1\end{array}\right), \mathrm{X}=\left\{\mathrm{x}_{t}^{\tilde{\alpha}_{M S}}\right\}, \mathrm{x}_{t}^{\tilde{\alpha}_{M S}}=\left(1-\tilde{\alpha}_{M S} L\right) \mathrm{x}_{t}(t=2, \ldots, T), \mathrm{x}_{1}^{\tilde{\alpha}_{M S}}=\mathrm{x}_{1}$ e $\hat{h}_{v}$ é uma estimativa de ( $2 \pi$ vezes) a função densidade espectral na frequência zero de $v_{t}$. Quando $\left|\widetilde{\alpha}_{M S}\right|<1$, o seguinte estimador baseado em Kernel é usado:

$$
\hat{h}_{v}=T^{-1} \sum_{t=1}^{T} \hat{v}_{t}^{2}+T^{-1} \sum_{j=1}^{T-1} \omega(j, m) \sum_{t=j+1}^{T} \hat{v}_{t} \hat{v}_{t-j}
$$

em que $\hat{v}_{t}$ são os resíduos $\mathrm{MQO}$ da regressão da equação 4 . A função $\omega(j, m)$ é o Kernel espectral quadrático e o bandwidth $m$ é selecionado de acordo com o método plug-in usando uma aproximação AR(1), como proposto por Andrews (1991).

Para o caso de erros I(1), Perron e Yabu (2009) propõem a seguinte estatística de Wald:

$$
W_{R Q F}^{*}\left(\lambda_{1}\right)=\left[\mathrm{R}\left(\tilde{\Psi}^{*}-\Psi\right)\right]^{\prime}\left[\hat{h}_{v} \mathrm{R}\left(\mathrm{X}^{\prime} \mathrm{X}\right)^{-1} \mathrm{R}^{\prime}\right]^{-1}\left[\mathrm{R}\left(\tilde{\Psi}^{*}-\Psi\right)\right]
$$

em que $\tilde{\Psi}=\left(\tilde{\mu}_{0}^{F G}, \tilde{\beta}_{0}^{F G}, \mu_{1}^{*}, \tilde{\beta}_{1}^{F G}\right)^{\prime}, \mu_{1}^{*}=\hat{h}^{1 / 2} \hat{\zeta}(L) \tilde{\mu}_{1}^{F G}\left(T_{1}\right) / \hat{\sigma}_{e k}, \tilde{\mu}_{1}^{F G}\left(T_{1}\right)$ é uma sequência de estimativas de $\tilde{\mu}_{1}^{F G}$ para diferentes valores da data da quebra $T_{1}\left(=\lambda_{1} \mathrm{~T}\right)$, e $\hat{\zeta}(L)$ e $\hat{\sigma}_{e k}$ é obtido da regressão

$$
\hat{v}_{t}=\sum_{i=1}^{k} \zeta_{i} \hat{v}_{t-i}+e_{i k}
$$

8 Perron e Yabu (2009) usam $a=10$ porque isso implica em testes com melhores propriedades. 
na qual $\hat{\zeta}(L)=\left(1-\hat{\zeta}_{1} L-\ldots-\hat{\zeta}_{k} L^{k}\right)$ e $\hat{\sigma}_{e k}=\left[(T-k)^{-1} \sum_{t=k+1}^{T} \hat{e}_{t k}^{2}\right]^{1 / 2}$. O critério de informação BIC é mais uma vez usado para a determinação dos termos autorregressivos incluídos na equação 8.

Perron e Yabu (2009) observam que o funcional Exp do teste de Wald para diferentes datas da quebra, dado por

$$
\operatorname{Exp} W=\log \left[T^{-1} \sum_{\lambda_{1} \in \Lambda_{1}} \exp \left(\frac{1}{2} W_{R Q F}\left(\lambda_{1}\right)\right)\right]
$$

têm distribuições limite quase idênticas nos casos de erros I(0) e I(1). Em razão disso, essa estatística é utilizada para testar a hipótese nula de uma quebra estrutural na função tendência considerando a data da quebra desconhecida a priori. ${ }^{9}$

Baseado em Perron e Yabu (2009), Kejriwal e Perron (2010) apresentam um procedimento sequencial que possibilita obter uma estimativa do número de quebras na função tendência independente se os erros são estacionários ou integrados. A primeira etapa desse procedimento consiste em testar a hipótese nula de ausência de quebra na função tendência contra a hipótese alternativa de uma quebra. Se a hipótese nula é rejeitada, a estimativa da data da quebra é obtida através da minimização global da soma dos quadrados dos resíduos. ${ }^{10} \mathrm{O}$ passo seguinte é testar a presença de uma quebra estrutural em cada um dos dois segmentos delimitados pela data da quebra estimada no passo anterior. Se o máximo da estatística de teste ExpW for significativa, então rejeita-se a hipótese nula de uma quebra em favor da hipótese alternativa de duas quebras. Então, as datas das duas quebras são estimadas através da minimização global da soma dos quadrados dos resíduos, e procede-se novamente com os testes de uma quebra adicional em cada um dos segmentos obtidos. Por exemplo, se desejamos testar a hipótese nula de uma quebra contra a hipótese alternativa de duas quebras, usamos a estatística de teste

$$
\operatorname{Exp} W(2 \mid 1)=\max _{1 \leq i \leq 2}\left\{\operatorname{Exp} W^{(i)}\right\}
$$

na qual ExpW $W^{(i)}$ é a estatística de teste para uma quebra no segmento $i$.

\subsection{Testes de Raiz Unitária}

Para investigar a ordem de integração da razão consumo-renda, aplica-se primeiramente o teste ADF. Entretanto, esse e outros testes normalmente empre-

9 Os valores críticos são apresentados em Perron e Yabu (2009).

10 Ver Bai e Perron (2003) para detalhes sobre a estimação das datas das quebras. 
gados pela literatura possuem um baixo poder quando há quebras estruturais no nível e/ou tendência da série temporal. Diante disso, utilizam-se os testes de raiz unitária propostos por Carrion-i-Silvestre et al. (2009). Esses autores propõem uma extensão da classe de testes M, analisada por Ng e Perron (2001), que permite múltiplas quebras estruturais no nível e na inclinação da função tendência sob as hipóteses nula e alternativa. Para uma breve descrição desses testes, considera-se um modelo com duas quebras no nível e na inclinação da função tendência:

$$
y_{t}=\mu_{0}+\mu_{1} D U_{1 t}+\mu_{2} D U_{2 t}+\beta_{0} t+\beta_{1} D T_{1 t}+\beta_{2} D T_{2 t}+u_{t}
$$

em que $D U_{i t}=\mathrm{I}\left(t>T_{i}\right)$ e $D T_{i t}=\mathrm{I}\left(t>T_{i}\right)\left(t-T_{i}\right)$, para $i=1$, 2. Posto isso, as estimativas das frações das quebras $\lambda=\left(\lambda_{1}, \lambda_{2}\right)$ e dos parâmetros do modelo, $\Psi=\left(\mu_{0}\right.$, $\mu_{1}, \mu_{2}, \beta_{0}, \beta_{1}, \beta_{2}$ ), são obtidas através da minimização da soma dos quadrados dos resíduos da regressão quase diferenciada. ${ }^{11} \mathrm{O}$ passo seguinte consiste na estimação por MQO da seguinte regressão:

$$
\Delta \tilde{y}_{t}=b_{0} \tilde{y}_{t-1}+\sum_{j=1}^{k} b_{j} \Delta \tilde{y}_{t-j}+e_{t k}
$$

com

$$
\tilde{y}_{t}=y_{t}-\hat{\Psi}^{\prime} \mathrm{x}_{t}(\hat{\lambda}), \mathrm{x}_{t}(\hat{\lambda})=\left\{1, D U_{1 t}(\hat{\lambda}), D U_{2 t}(\hat{\lambda}), D T_{1 t}(\hat{\lambda}), D T_{2 t}(\hat{\lambda})\right\}
$$

e obtenção de uma estimativa autorregressiva da densidade espectral de $v_{t}$ na frequência zero, dada por

$$
s^{2}(\hat{\lambda})=s_{e k}^{2} /(1-\hat{b}(1))^{2}
$$

na qual $s_{e k}^{2}=(T-k)^{-1} \sum_{t=k+1}^{T} \hat{e}_{t k}^{2}$ e $\hat{b}(1)=\sum_{j=1}^{k} \hat{b}_{j}$. Com base nisso, as seguintes extensões da classe de testes $\mathrm{M}$ de raiz unitária são consideradas:

$$
\begin{gathered}
M Z_{a}^{G L S}(\hat{\lambda})=\left(T^{-1} \tilde{y}_{T}^{2}-s^{2}(\hat{\lambda})\right)\left(2 T^{-2} \sum_{t=1}^{T} \tilde{y}_{t-1}^{2}\right)^{-1} \\
M Z_{t}^{G L S}(\hat{\lambda})=\left(T^{-1} \tilde{y}_{T}^{2}-s^{2}(\hat{\lambda})\right)\left(4 s^{2}(\hat{\lambda}) T^{-2} \sum_{t=1}^{T} \tilde{y}_{t-1}^{2}\right)^{-1 / 2} \\
M P_{T}^{G L S}(\hat{\lambda})=\left[\bar{c}^{2} T^{-2} \sum_{t=1}^{T} \tilde{y}_{t-1}^{2}+(1-\bar{c}) T^{-1} \tilde{y}_{T}^{2}\right] / s^{2}(\hat{\lambda})
\end{gathered}
$$

$11 \quad$ Para detalhes, ver Carrion-i-Silvestre et al. (2009). 
A realização dos testes de raiz unitária exige a seleção de um número $k$ de defasagens ótimas dos termos de diferença defasados. Segundo Ng e Perron (2001), critérios de seleção tais como o BIC ou o critério de informação de Akaike (AIC) tendem a fornecer valores de $k$ muito pequenos para que o teste de estacionariedade tenha uma dimensão adequada. Conforme sugerido pelos autores, o critério de informação adotado pela presente análise foi o Akaike modificado (MAIC), tanto na implementação do ADF quanto nos testes de Carrion-i-Silvestre et al. (2009). Adicionalmente, é importante ressaltar, ainda, que os testes de raiz unitária com quebras estruturais devem ser realizados apenas para os casos em que a série temporal apresenta quebras estruturais na função tendência. Isso ocorre porque esses testes apresentam um baixo poder se a série temporal não apresenta quebras estruturais. Assim, se a PMC não apresenta quebras estruturais, verifica-se a ordem de integração dessa variável através da classe de testes M de raiz unitária apresentados por Ng e Perron (2001).

\section{Resultados Empíricos}

\subsection{Dados}

O conjunto de dados foi extraído da Penn World Table, versão 7.1, e se referem à renda anual (RGDPL) e à razão consumo-renda anual (PMC) para o período de 1951 a 2010. ${ }^{12}$ Os países analisados são: Argentina, Bolívia, Brasil, Chile, Colômbia, Equador, Paraguai, Peru, Uruguai, Venezuela, México e Estados Unidos. Para a aplicação dos testes, considera-se o logaritmo natural da PMC de cada país.

A Figura 1 mostra a evolução da PMC e da renda, bem como a do consumo agregado. A escala do eixo esquerdo corresponde aos valores da renda e do consumo, enquanto que a escala do eixo direito está relacionada aos valores da razão consumo-renda. A análise visual sugere que a PMC de alguns países apresentou importantes mudanças em suas dinâmicas. Aparentemente, a maioria das quebras ocorreu nas décadas de 1960 e 1970. Nesse período, observam-se fortes quedas no nível da PMC para Argentina, Brasil, Equador, Paraguai e Uruguai. Em contraposição, o Chile apresentou uma importante elevação da razão consumo-renda na década de 1960. Nos anos de 1980, a PMC nas economias da Bolívia e do Brasil parece apresentar uma quebra estrutural positiva.

12 Em específico, a variável renda é o PIB per capita em paridade do poder de compra (PPP) a preços constantes de 2005, e a PCM é a participação do consumo das famílias nessa renda. 
Figura 1 - Evolução da PMC, consumo e renda

Argentina

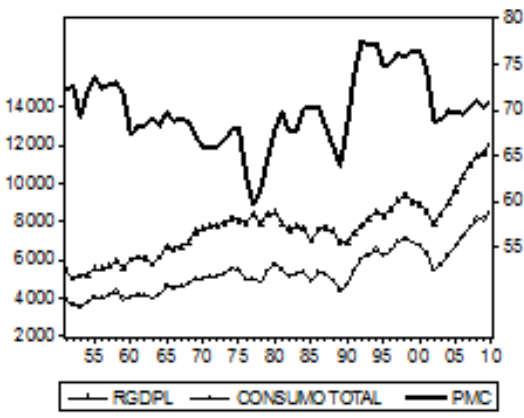

Brasil
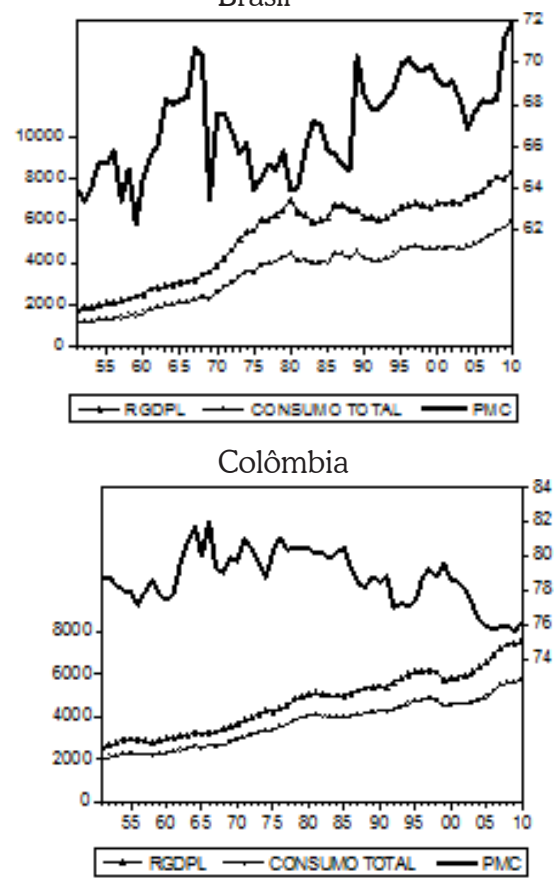

Estados Unidos
Bolívia

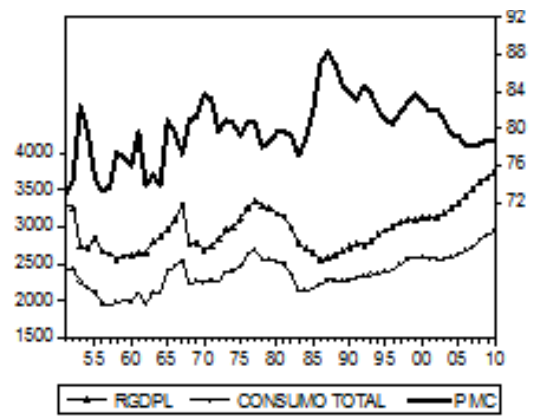

Chile
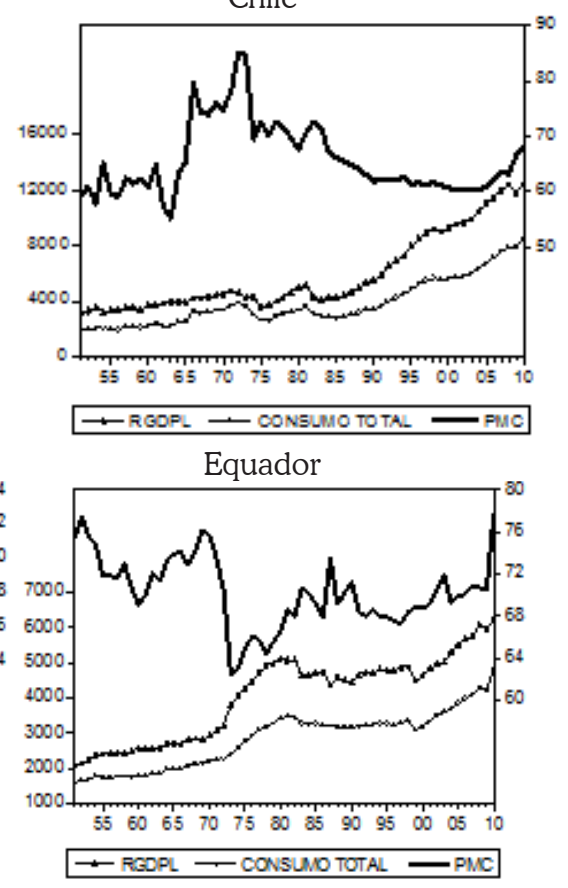

México 


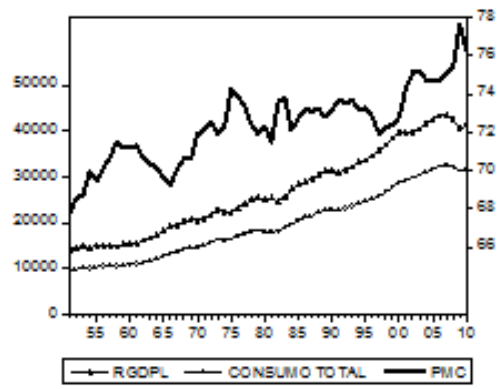

Paraguai
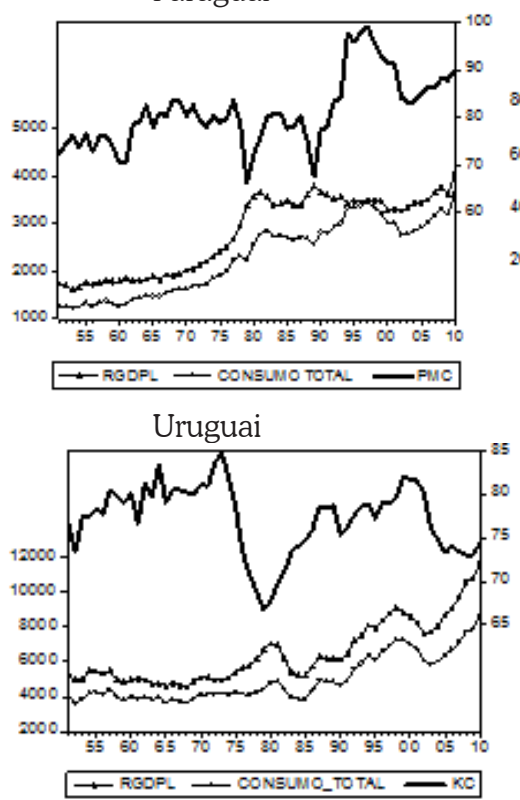

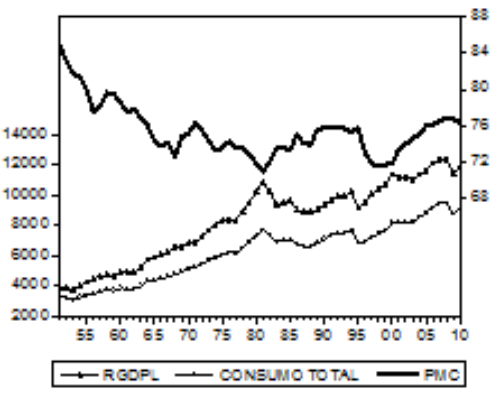

Peru
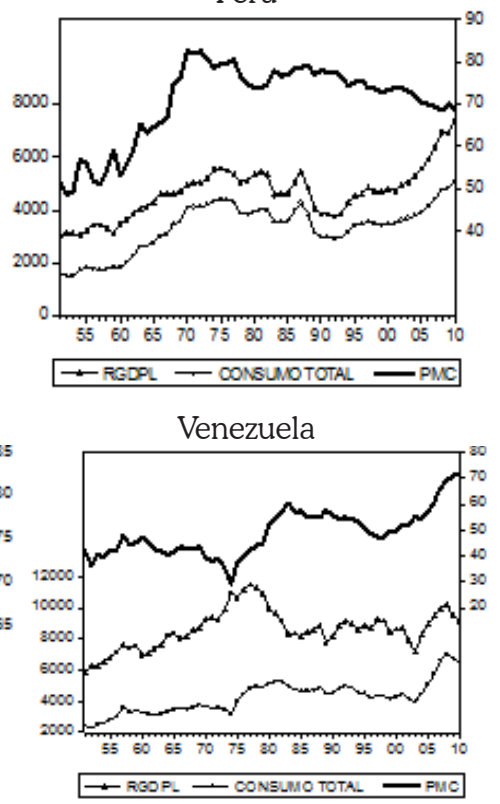

Fonte: Elaboração própria.

Gomes e Franchini (2009) destacam a influência de choques externos nos padrões exibidos pelas séries ao longo do tempo. Os autores elegem três possíveis choques principais: os dois choques no preço do petróleo e a crise da dívida em algumas economias. Nos anos de 1973 a 1974, a Organização dos Países Exportadores de Petróleo (Opep) promoveu a primeira redução na oferta do produto, gerando um aumento substancial nos preços, o que produziu alta inflação em países emergentes e desenvolvidos. De 1978 a 1979, a ocorrência de um segundo choque de oferta desencadeou os mesmos efeitos observados anteriormente. A elevação dos preços do petróleo e, mais adiante, a crise da dívida afetaram nega- 
tivamente os déficits dos países latino-americanos, dificultando o pagamento das obrigações aos credores. ${ }^{13}$

Nos anos 2000, verifica-se que poucos países exibiram mudanças abruptas na PMC. Em meados de 2001, a Argentina apresentou uma forte queda na razão consumo-renda. Nesse mesmo período houve no país o decreto de calote à dívida pública e queda no produto da economia. Depois de uma quebra negativa na segunda metade da década de 1990, a PMC do Brasil voltou ao seu padrão de crescimento no início dos anos 2000, provavelmente em razão do crescimento do consumo estimulado pelas políticas de incentivo do governo. Para os Estados Unidos, nota-se uma clara tendência de crescimento na sua razão consumo-renda da segunda metade da década de 1990 até 2008, seguida de uma queda no biênio 2009/2010. Uma possível explicação para essa redução foi a crise econômica que atingiu a economia norte-americana nesse período e impactou mais fortemente $o$ consumo das famílias do que o produto agregado.

\subsection{Quebras Estruturais e Testes de Raiz Unitária}

Esta seção traz os resultados dos testes que compõem a estratégia empírica explanada na seção anterior. Inicialmente são procedidos os testes para quebras estruturais a fim de verificar se as séries analisadas podem ser caracterizadas por mudanças nos coeficientes da função tendência. Os resultados desses testes são apresentados na Tabela $1 .{ }^{14}$

13 Gomes e Franchini (2009), seguindo Ben-David e Loewy (1998) e Ferreira et al. (2010), analisam a distribuição das quebras, tendo em mente a ocorrência de diversos fatos históricos. Os autores atentam para o fato desse exercício não representar uma relação de causalidade.

14 Utilizou-se o software Gauss 9.0 para se realizar as estimações. 
Tabela 1 - Testes para quebras estruturais na função tendência da PMC

\begin{tabular}{lcccc}
\hline \multirow{2}{*}{ País } & \multicolumn{2}{c}{ Estatística $\mathbf{W}_{\mathbf{R Q F}}$} & Primeira quebra & Segunda quebra \\
\cline { 2 - 5 } & $\mathbf{( 1 | 0 )}$ & $\mathbf{( 2 | 1 )}$ & Ano & Ano \\
\hline Argentina & 1,429 & $23,65 * * *$ & 1979 & 1990 \\
Bolívia & 1,907 & $9,402 * * *$ & 1964 & 1985 \\
Brasil & $8,708^{* * *}$ & $37,19 * * *$ & 1968 & 1988 \\
Chile & $5,946^{* * *}$ & $100,9 * * *$ & 1965 & 2001 \\
Colômbia & $2,716^{* *}$ & 0,372 & 1962 & - \\
Equador & $8,214^{* * *}$ & $5,058^{* * *}$ & 1972 & 1990 \\
México & $3,786^{* *}$ & $11,59 * * *$ & 1982 & 1995 \\
Paraguai & $4,097 * *$ & $38,76^{* * *}$ & 1978 & 1991 \\
\hline Peru & $102,8^{* * *}$ & 0,523 & 1967 & - \\
Estados & 1,193 & 1,948 & - & - \\
Unidos & & & & \\
Uruguai & 0,566 & $7,072 * * *$ & 1975 & 2001 \\
Venezuela & $7,935^{* * *}$ & $6,433 * * *$ & 1979 & 1996 \\
\hline
\end{tabular}

Fonte: Elaboração própria.

Nota: Os símbolos *, ** e *** denotam significância estatística a 10\%, 5\% e 1\%, respectivamente.

A primeira fase do procedimento de Kejriwal e Perron (2010) testa a hipótese nula de zero quebra versus a hipótese alternativa de uma quebra no nível e na inclinação da função tendência da PMC. Para um nível de significância de 5\%, os resultados da Tabela 1 indicam a rejeição da hipótese de ausência de quebra na tendência da PMC para as economias do Brasil, Chile, Colômbia, Equador, México, Paraguai, Peru e Venezuela. Em contraposição, os testes indicam a ausência de quebra estrutural nos coeficientes da função tendência da razão consumo-renda na Argentina, Bolívia, Estados Unidos e Uruguai.

Em razão do tamanho da amostra, procura-se testar a existência de apenas uma quebra adicional na função tendência da razão consumo-renda. Embora o valor da estatística $W_{R Q F}(1 \mid 0)$ não seja significativa para alguns países, testa-se a hipótese nula de uma quebra versus a hipótese alternativa de duas quebras na função tendência da PMC em todas as economias analisadas. Isso é feito porque os testes para uma única quebra podem sofrer de baixo poder em amostras finitas na presença de múltiplas quebras (PRODAN, 2008). Conforme os resultados mostrados na Tabela 1, apenas a Colômbia e o Peru possuem só uma quebra estrutural, ocorrida nos anos de 1962 e 1967, respectivamente. Nos casos da Argentina, Bolívia e Uruguai, cujos testes iniciais sugeriram que os parâmetros da função 
tendência eram constantes, rejeita-se a hipótese de uma quebra em favor de duas quebras a um nível de significância de 1\%. Dado a possibilidade de baixo poder do teste $W_{R Q F}(1 \mid 0)$, considerou-se a presença de duas quebras estruturais na função tendência da PMC daquelas economias. Por fim, os resultados dos testes para a economia norte-americana indicam que os coeficientes da função tendência mantiveram-se estáveis ao longo do período estudado.

As estimativas das datas das quebras estruturais na função tendência da razão consumo-renda são apresentadas na Tabela 1 e ilustradas na Figura 2. Com exceção do México, todos os países apresentaram a primeira quebra nas décadas de 1960 e 1970, fato que pode estar associado aos choques externos (tais como os choques do petróleo) e a choques internos peculiares a cada país. As segundas quebras aconteceram, em geral, no intervalo de 1985 a 2001. Por exemplo, o Brasil e a Bolívia exibiram uma segunda quebra ainda na década de 1980, enquanto a Argentina, Equador, Paraguai, México e Venezuela apresentaram mais uma quebra na década de 1990; o Chile e o Uruguai apresentaram no ano de 2001. As quebras ocorridas na segunda metade da década de 1980 e no início da de 1990 podem estar associadas aos reflexos da instabilidade econômica que afetou negativamente vários países latino-americanos nesse período. Em alguns países, os choques internos também podem estar relacionados a essas quebras estruturais. Por exemplo, o México foi afetado por uma forte crise financeira em 1994. Na Venezuela, o início dos anos de 1990 foi marcado por uma conjuntura de instabilidade econômica, resultante da combinação de choques externos e internos, como a queda do preço do barril de petróleo e a crise bancária de 1994.

Figura 2 - Evolução da PMC, da função tendência e datas das quebras
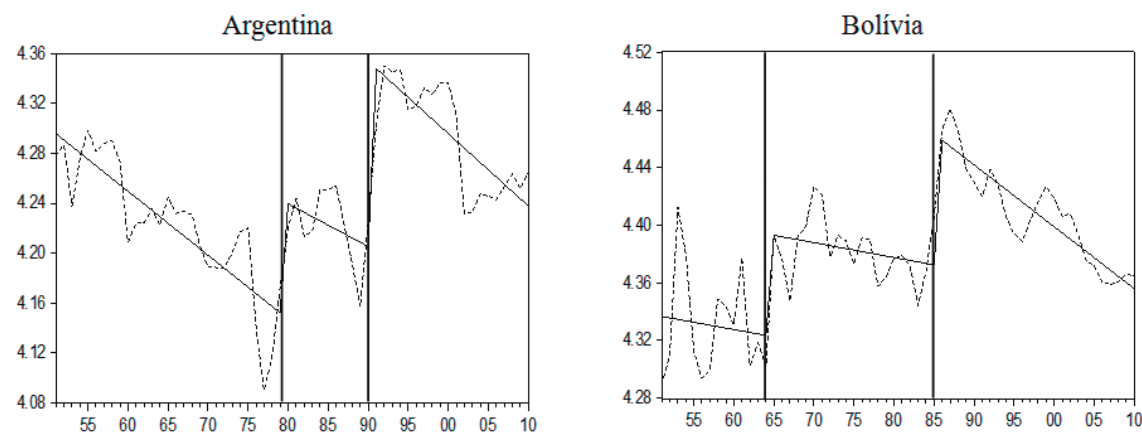
Brasil

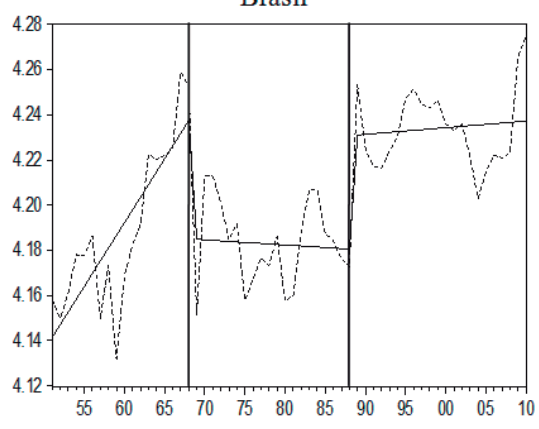

Colômbia

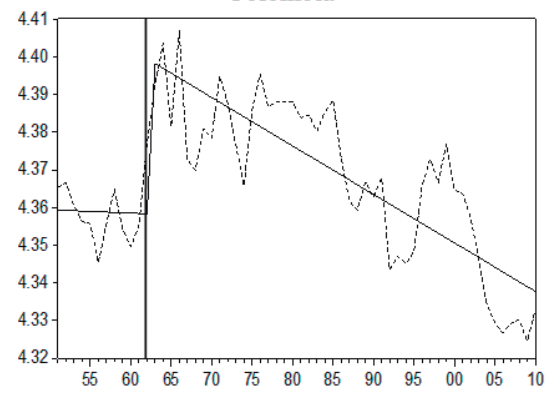

Estados Unidos

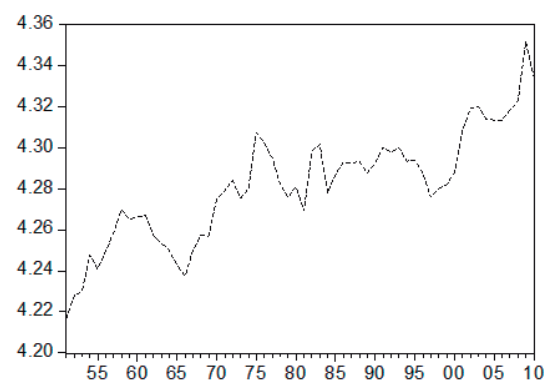

Paraguai

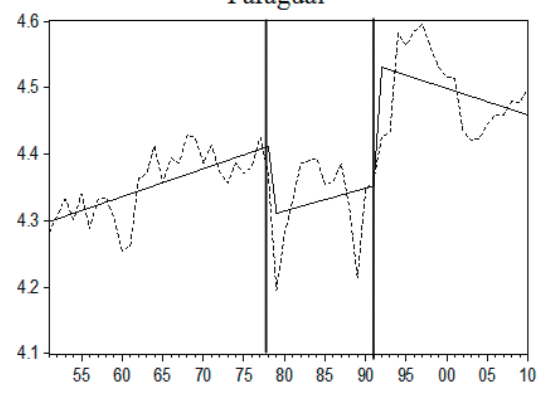

Chile

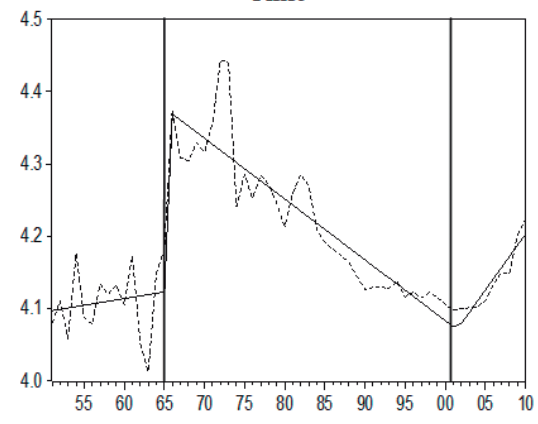

Equador
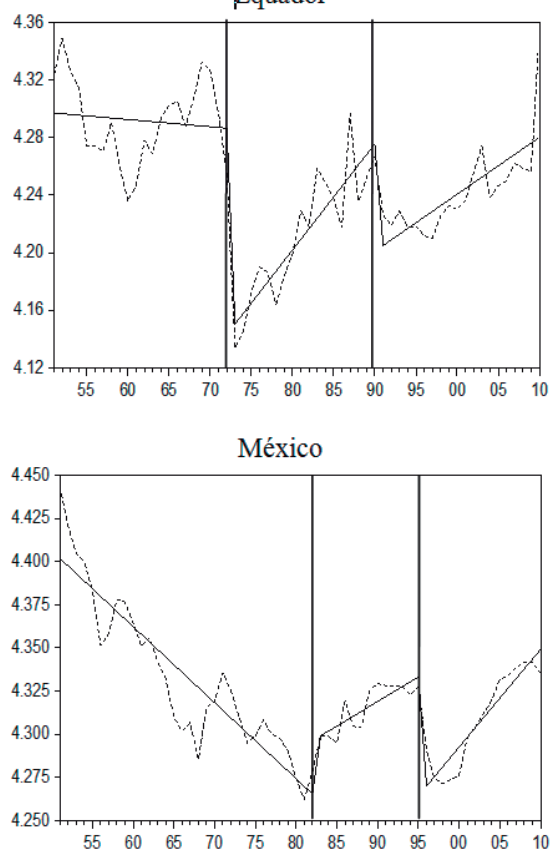

Peru

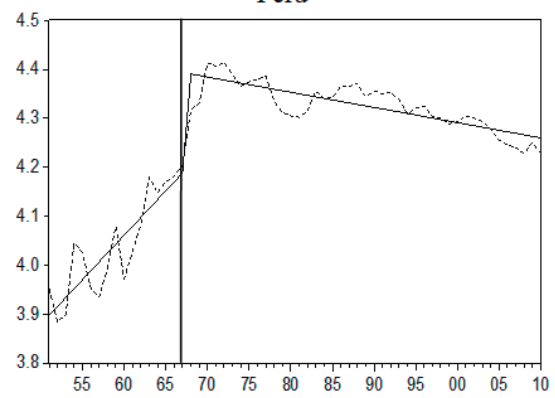



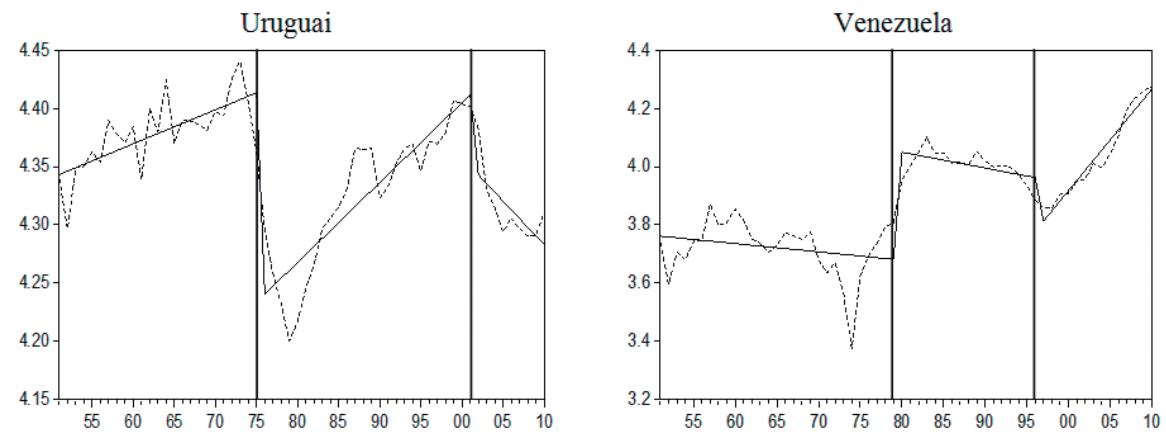

Fonte: Elaboração própria.

É interessante comparar os resultados deste trabalho com os obtidos por Gomes e Franchini (2009). Esses autores realizam testes de raiz unitária supondo a existência de duas quebras estruturais na função tendência da PMC dos países da América do Sul, com exceção da Argentina e do Uruguai, os quais apresentam uma quebra estrutural que foi considerada. Em contraposição, os resultados dos testes $W_{R Q F}$ mostrados na Tabela 1 indicam a existência de duas quebras estruturais na função tendência da PMC da Argentina e do Uruguai, e de apenas uma quebra na PMC da Colômbia e do Peru. Adicionalmente, as estimativas das datas das quebras obtidas por Gomes e Franchini (2009) para Bolívia (1969 e 1984), Brasil (1983 e 1998), Chile (1964 e 1974), Colômbia (1978 e 1997), Equador (1971 e 1985), Paraguai (1965 e 1978) e Venezuela (1968 e 1981) apresentaram algumas diferenças importantes das mostradas anteriormente. Essas divergências podem ser decorrentes: a) de mudanças metodológicas realizadas pela Penn World Table, entre os anos de 2006 e 2012, no cálculo do consumo das famílias e, consequentemente, nas séries da PMC; $;{ }^{15}$ b) de uso de diferentes estimadores para as datas das quebras $;{ }^{16} \mathrm{c}$ ) do maior número de observações usadas neste trabalho, que permitiu identificar períodos de quebras mais recentes, tais como as quebras observadas em 2001 para a PMC do Chile e do Uruguai.

A Tabela 2 traz os resultados dos testes de raiz unitária para a razão consumo-renda. Para os Estados Unidos, a classe de testes M sugerido por Ng e Perron

15 Por exemplo, na Penn World Table, versão 7.1, o conceito de consumo inclui os gastos com consumo das famílias mais a provisão de serviços às famílias por instituições não lucrativas e pelo governo (principalmente, os gastos com educação e saúde). Por outro lado, a Penn World Table versão 6.2, usada por Gomes e Franchini (2009), define o consumo apenas como os gastos com consumo das famílias. Para detalhes, ver Heston et al. (2012).

16 Gomes e Franchini (2009) seguem Lee e Strazicich (2003) e usam um procedimento de estimar as datas das quebras através da minimização de testes de raiz unitária sobre todas as possíveis datas das quebras. Entretanto, evidências de simulação apresentadas por Vogelsang e Perron (1998) e Lee e Strazicich (2001) sugerem que esse procedimento é improvável de prover estimativas consistentes das verdadeiras datas das quebras. 
(2001) é utilizada porque não se encontrou evidência de quebra estrutural na função tendência da PMC. Já para os demais países, os testes M são aqueles descritos na seção 2.2 e consideram múltiplas quebras no nível e na inclinação da função tendência.

Tabela 2 - Testes de raiz unitária

\begin{tabular}{lccccc}
\hline País & ADF & Quebras & $\mathbf{M Z}_{\alpha}$ & $\mathbf{M Z}_{\mathbf{t}}$ & $\mathbf{M P}_{\mathbf{T}}$ \\
\hline Argentina & $-2,296$ & 2 & $-17,42$ & $-2,940$ & 12,93 \\
Bolívia & $-1,404$ & 2 & $-21,82$ & $-3,299$ & 10,87 \\
Brasil & $-2,554$ & 2 & $-19,15$ & $-2,984$ & 13,09 \\
Chile & $-2,476$ & 2 & $-5,258$ & $-1,307$ & 33,38 \\
Colômbia & $-2,042$ & 1 & $-12,18$ & $-2,453$ & 13,46 \\
Equador & $-2,011$ & 2 & $-19,07$ & $-2,911$ & 13,31 \\
Estados & $-3,393$ & 0 & $-13,04$ & $-2,547$ & 7,023 \\
Unidos & & & & & \\
México & $-2,621$ & 2 & $-13,09$ & $-2,554$ & 16,18 \\
Paraguai & $-3,065$ & 2 & $-19,22$ & $-3,085$ & 11,38 \\
Peru & $-1,273$ & 1 & $-20,89^{*}$ & $-3,200^{*}$ & $8,089^{*}$ \\
Uruguai & $-2,076$ & 2 & $-11,21$ & $-2,346$ & 20,55 \\
Venezuela & $-1,948$ & 2 & $-12,31$ & $-2,449$ & 18,49 \\
\hline
\end{tabular}

Fonte: Elaboração própria.

Nota: O símbolo * denota significância estatística a 10\%.

A aplicação do teste $\mathrm{ADF}$ mostra que a hipótese nula de raiz unitária da PMC pode ser rejeitada em todas as séries testadas. No entanto, conforme já explicitado, esse teste possui um baixo poder na presença de mudanças estruturais na série, de modo que a sua utilização consiste em uma referência para comparação com os demais testes. Para a classe de testes M, observa-se que apenas a série pertencente ao Peru apresentou rejeição da hipótese de raiz unitária para um nível de significância de $10 \%$. Para os demais países, os resultados indicam que a PMC é não estacionária.

Em geral, os resultados acima são diferentes dos apresentados por Cook (2005) e Gomes e Franchini (2009). Esses autores usam o teste LM mínimo de raiz unitária e concluem que a razão consumo-renda dos Estados Unidos e dos países da América do Sul (com exceção do Uruguai) é estacionária. No presente trabalho, a utilização de uma amostra mais recente, o uso de novos procedimentos para testar a existência e estimar as datas das quebras estruturais na função tendência, e o emprego de diferentes testes de raiz unitária em conformidade com o número de 
quebras observadas podem explicar as divergências de resultados. Vale ressaltar ainda que as evidências da não estacionariedade da PMC estão em consonância com as constatações empíricas apresentadas por Cerrato et al. (2013), que empregam testes de raiz unitária para painel de dados que permitem não linearidades e mudanças abruptas.

\section{Considerações Finais}

Neste trabalho, verificou-se o comportamento da razão consumo-renda em 11 países da América Latina, além dos Estados Unidos, no período de 1951-2010. Para isso, utilizaram-se os procedimentos propostos por Perron e Yabu (2009) e Kejriwal e Perron (2010) a fim de se verificar a existência e o número de quebras estruturais no nível e inclinação da função tendência das séries. Feito isso, averiguou-se a estacionariedade da razão consumo-renda através da aplicação da classe de testes M de raiz unitária apresentada por Carrion-i-Silvestre et al. (2009). Esses testes permitem múltiplas quebras estruturais na função tendência sob as hipóteses nula e alternativa.

O teste para quebras estruturais na função tendência da PMC indicou a existência de duas quebras para a maior parte das economias analisadas, entre elas as da Argentina, Brasil, Paraguai e Uruguai. As exceções foram a Colômbia e o Paraguai, que apresentaram apenas uma quebra. As datas estimadas para as quebras concentraram-se especialmente nas décadas de 1970 e 1990, períodos marcados por choques externos e internos ligados a eventos históricos, tais como a alta no preço do petróleo, crise da dívida, hiperinflação e mudanças na condução de política econômica. Para os Estados Unidos, não se pôde rejeitar a hipótese de ausência de quebras estruturais dos parâmetros da função tendência da PMC.

Com relação à ordem de integração da razão consumo-renda, os resultados dos testes de raiz unitária indicaram que a maior parte das séries estudadas não é estacionária. As evidências de não rejeição da hipótese nula de raiz unitária foram encontradas nas séries da PMC de países como Argentina, Bolívia, Brasil, Chile, Colômbia, Equador, Paraguai, Uruguai e Venezuela. O México também se enquadra nessa categoria, ao lado dos Estados Unidos, para o qual a classe de teste $\mathrm{M}$ de raiz unitária apontou para a não estacionariedade da razão consumo-renda. A única exceção foi o Peru, no qual a hipótese de raiz unitária na PMC foi rejeitada a um nível de significância de $10 \%$.

Faz-se mister destacar que os resultados apontando para a não estacionariedade da razão consumo-renda têm importantes implicações teóricas e empíricas. Primeiro, eles são favoráveis à teoria keynesiana da renda absoluta, mas estão em desconformidade com teorias que predizem a suavização do consumo das famílias e estabilidade da PMC, tais como o modelo de formação de hábito, a hipótese da 
renda permanente e a hipótese do ciclo da vida. A ausência de suporte empírico para essas teorias pode ser decorrente da existência de restrições à tomada de empréstimos e/ou à presença de consumidores míopes ou keynesianos nas economias analisadas. ${ }^{17}$ Segundo, os resultados não suportam a predição teórica do modelo de crescimento neoclássico estocástico e de outros modelos de crescimento de que a razão consumo-renda seria estacionária na trajetória de crescimento equilibrado..$^{18}$ Finalmente, os resultados observados implicam que choques temporários exercem um efeito permanente sobre a razão consumo-renda dos países estudados. Nesse caso, a modelagem empírica de modelos teóricos envolvendo a PMC deve testar se as variáveis do sistema são cointegradas a fim de evitar o problema de regressão espúria.

\section{Referências}

ANDO, A.; MODIGLIANI, F. The life cycle hypothesis of saving: aggregate implications and tests. American Economic Review, v. 53, n. 1, p. 55-84, 1963.

ANDREWS, D. W. K. Heteroskedasticity and autocorrelation consistent covariance matrix estimation. Econometrica, v. 59, n. 3, p. 817-858, 1991.

BAI, J.; PERRON, P. Computation and analysis of multiple structural change models. Journal of Applied Econometrics, v. 18, n. 1, p. 1-22, 2003.

BEN-DAVID, D.; LOEWY, M. Free trade growth and convergence. Journal of Economic Growth, v. 3, n. 2, p. 143-170, 1998.

CAMPBELL, J. Y.; PERRON, P. Pitfalls and opportunities: what macroeconomists should know about unit roots. NBER Macroeconomics Annual, v. 6, p. 141-220, 1991.

CAMPBELL, J.; MANKIW, N. G. Permanent income, current income, and consumption. Journal of Business and Economics Statistics, v. 8, n. 3, p. 265-279, 1990.

CAMPBELL, J.Y. Does saving anticipate declining labor income? an alternative test of the permanent income hypothesis. Econometrica, v. 55, n. 6, p. 1249-1273, 1987.

CARRION-I-SILVESTRE, J.L.; KIM, D.; PERRON, P. GLS-based unit root tests with multiple structural breaks both under the null and the alternative hypotheses. Econometric Theory, v. 25, n. 6, p. 1754-1792, 2009.

CERRATO, M.; PERETTI, C.; STEWART, C. Is the consumption-income ratio stationary? Evidence from linear and non-linear panel unit root tests for OECD and non-OECD countries. The Manchester School, v. 81, n. 1, p. 102-120, 2013.

COOK, S. The stationary of consumption-income ratios: evidence from minimum LM unit root testing. Applied Economics Letters, v. 89, n.1, p. 55-60, 2005.

17 Sobre a presença de restrição de liquidez e/ou consumidores míopes para algumas das economias analisadas neste trabalho, ver, por exemplo, Campbell e Mankiw (1990), Shea (1995a, 1995b), Gomes e Paz (2004, 2010) e Gomes (2010).

18

Ver, por exemplo, King et al. (1991) e Lau e Sin (1997). 
DICKEY, D.; FULLER, W. Distribution of the estimators for autoregressive time series with a unit root. Journal of the American Statistical Association, v. 74, n. 366, p. 427-431, 1979.

DROBNY, A.; HALL, S. An investigation of the long-run properties of aggregate nondurable consumers expenditure in the united kingdom. Economic Journal, v. 99, n. 396, 1989.

DUESENBERRY, J. S Income, saving and the theory of consumer behavior. Cambridge: Harvard University Press, 1952.

FALLAHI, F. The stationarity of consumption-income ratios: Evidence from bootstrapping confidence intervals. Economics Letters, v. 115, n. 1, p. 137-140, 2012.

FERREIRA, P. C. et al. The effects of external and internal shocks on total factor productivity. The Quarterly Review of Economics and Finance v. 50, n. 3, p. 298-309, 2010.

FRIEDMAN, M. A. A theory of the consumption function. Princeton: Princeton University Press, 1995.

GOMES, F. A. R. Consumo no Brasil: comportamento otimizador, restrição de crédito ou miopia? Revista Brasileira de Economia, v. 64, n. 3, p. 261-275, 2010.

GOMES, F. A. R.; FRANCHINI, D. S. The stationarity of consumption-income ratios: evidence from south American countries. Economia Aplicada, v. 13, n. 4, p. 463-479, 2009.

GOMES, F. A. R.; PAZ, L. Especificações para a função consumo: testes para países da América do Sul. Pesquisa e Planejamento Econômico, v. 34, n. 1, p. 39-55, 2004.

GOMES, F.; PAZ, L. Consumption in South America: myopia or liquidity constraints? Economia Aplicada, v. 14, n. 2, p. 129-145, 2010.

HALL, S.; PATTERSON, K. A systems approach to the relationship between consumption and wealth. Applied Economics, v. 24, n. 10, p. 1165-1171, 1992.

HESTON, A.; SUMMERS, R.; ATEN, B. Penn World Table Version 7.1. University of Pennsylvania: Center for International Comparisons of Production, Income and Prices, 2012. (Mimeo).

HORIOKA, C. A cointegration analysis of the impact of the age structure of the population on the household saving rate in Japan. Review of Economic and Statistics, v. 79, n. 3, p. 511 $516,2000$.

KEJRIWAL, M.; PERRON, P. A sequential procedure to determine the number of breaks in trend with an integrated or stationary noise component. Journal of Time Series Analysis, v. 31, n. 5, p. 305-328, 2010.

KING, R.et al. Stochastic trends and economic fluctuations. American Economic Review, v. 81, n. 4, p. 819-840, 1991.

LAU, S-H. P.; SIN, C-Y. Observational equivalence and a stochastic cointegration test of the neoclassical and Romer's increasing returns models. Economic Modelling, v. 14, n. 1, p. 305-328. 1997.

LEE, J.; STRAZICICH, M. C. Break point estimation and spurious rejections with endogenous unit roots. Oxford Bulletin of Economics and Statistics, v. 63, n. 5, p. 535-558, 2001. 
. Minimum lagrange multiplier unit root test with two structural breaks. Review of Economics and Statistics, v. 85, n. 4, p. 1082-1089, 2003.

MARIOTT, F. H. C.; POPE, J. A. Bias in the estimation of auto-correlations. Biometrika, v. 41, n. 3/4, p. 390-342. 1954.

MOLANA, H. The time series consumption function: error correction, random walk and the steady state. Economic Journal, v. 101, n. 406, p. 382-403, 1991.

NG, S.; PERRON, P. Lag length selection and the construction of unit root tests with good size and power. Econometrica, v. 69, n. 6, p. 1519-1554, Nov. 2001.

PANTULA, S. G.; FULLER, W. A. Mean estimation bias in least squares estimation of autoregressive processes. Journal of Econometrics, v. 27, n. 1, p. 99-121, 1988.

PERRON, P. The great crash, the oil price shock, and the unit root hypothesis. Econometrica, v. 57, n. 6, p. 1361-1401, Nov. 1989.

PERRON, P.; YABU, T. Testing for shifts in trend with an integrated or stationary noise component. Journal of Business 8 Economic Statistics, v. 27, n. 3, p. 369-396, 2009.

PRODAN, R. Potential pitfalls in determining multiple structural changes with an application to Purchasing Power Parity. Journal of Business 8 Economic Statistics, v. 26, n. 1, p. 50-56, 2008.

ROY, A.; FULLER, W. A. Estimation for autoregressive processes with a root near one. Journal of Business 8 Economic Statistics, v. 19, n. 4, p. 482-493, 2001.

SARANTIS, N.; STEWART, C. Is the consumption-income ratio stationary? Evidence from panel unit root tests. Economics Letters, v. 64, n. 3, p. 309-314, Sept. 1999.

SHEA, J. Myopia, liquidity constraints, and aggregate consumption: A simple test. Journal of Money, Credit and Banking, v. 27, n. 3, p. 798-805, 1995a.

. Union contracts and the life cycle-permanent income hypothesis. American Economic

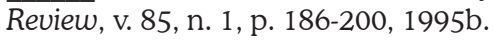

SLESNICK, D. T. Are our data relevant to the theory? The case of aggregate consumption. Journal of Business and Economic Statistics, v. 16, n. 1, p. 52-61, 1998.

TSIONAS, E.; CHRISTOPOULOS, D. Non-sationarity in the consumption? Income ratio: further evidence from panel and assymetric unit root tests. Economics Bulletin, v. 3, n. 12, p. $1-5,2002$.

UNGERN-STERNBERG, T. V. Inflation and the consumption function, Review of World Economics, v. 122, n. 4, p. 741-744, 1986.

VOGELSANG, T. J.; PERRON, P. Additional tests for a unit root allowing the possibility of breaks in the trend function. International Economic Review, v. 39, n. 4, p. 1073-1100, Nov. 1998.

Recebido em: 07/10/2013.

Aceito em: 13/03/2015. 